\title{
A State Estimation Method for Sound Environment Based on Fuzzy Observation
}

\author{
Akira Ikuta ${ }^{\dagger}$ \\ Faculty of Human Life \& Environmental Science, Hiroshima Prefectural Women's University, \\ 1-1-71 Ujina-Higashi, Minamiku, Hiroshima, 734-8558 Japan
}

Mitsuo Ohta ${ }^{\dagger}$

Hiroshima University, Emeritus, 1105, 1-7-10 Matoba, Hiroshima, 732-0824 Japan

\author{
M.O. Tokhi ${ }^{\dagger}$ \\ Department of Automatic Control \& Systems Engineering, The University of Sheffield, Mappin Street, Sheffield S1 3JD, UK
}

(Received 23 July 2001; accepted 17 December 2001)

\begin{abstract}
In the measurement of actual sound environments, the observed data exhibit various probability distribution forms, and often contain fuzziness due to confidence limitations in sensing devices, permissible errors in experimental data, and quantisation errors in digitised observations. In this study a fuzzy state estimation method is proposed on the basis of these fuzzy observations, from both the static and dynamic viewpoints. The validity and effectiveness of the proposed method are confirmed experimentally in the evaluation of road traffic noise.
\end{abstract}

${ }^{\dagger}$ Member of the International Institute of Acoustics and Vibration (IIAV)

\section{NOMENCLATURE}

$\langle x\rangle \quad$ - averaging operation with respect to $x$

$p(x) \quad$ - probability density function for a continuous variable $x$ or probability distribution for a discrete variable $x$

$p(x \mid M)$ - conditional probability density function for $x$ given that an event $M$ has taken place

$\langle x \mid M\rangle$ - conditional expectation with respect to $x$ given that an event $M$ has taken place

$\delta_{n m} \quad-$ Kronecker's delta

\section{INTRODUCTION}

The probability distribution of a specific signal in an actual sound environment can take various forms, not necessarily characterised by a standard Gaussian distribution. This is due to the diverse nature of factors affecting the properties of the signal. Therefore, it is necessary, for the estimation of the evaluation quantities such as $L_{x}, L_{e q}$, and the peak value of the signal, to consider the lower order statistical properties of the signal such as mean and variance as well as the higher order statistics associated with non-Gaussian properties.

On the other hand, the actual observed data often contain fuzziness due to confidence limitations in sensing devices, permissible errors in the experimental data, and quantisation errors in digital observations. For reasons of simplicity, many previously proposed estimation methods have not considered fuzziness in the observed data under the restriction of Gaussian type fluctuations. ${ }^{1-3}$ Although several state estimation methods for a stochastic environmental system with nonGaussian fluctuations have previously been proposed, ${ }^{4,5}$ the fuzziness contained in the observed data has not been considered in these studies. Therefore, it is desirable to develop a method that is flexible and is applicable to ill-conditioned fuzzy observations.
In this paper, a new estimation theory is proposed for a signal based on fuzzy observations with non-Gaussian properties, from both static and dynamic viewpoints. A static method for the estimation of the probability density function of the signal is proposed first using fuzzy probability. ${ }^{6} \mathrm{~A}$ dynamical state estimation method, that is flexible and applicable to fuzzy observations, is then obtained for a signal corrupted by additional noise (i.e., background noise) of nonGaussian type. Finally, the validity and effectiveness of the proposed method are confirmed experimentally with actual road traffic noise data.

\section{STATIC STATE ESTIMATION METHOD BASED ON FUZZY OBSERVATIONS}

Consider a sound environment system with an arbitrary distribution of non-Gaussian type. Using the statistical Hermite expansion, ${ }^{7,8}$ the probability density function of a specific signal $X$ in the sound pressure level can be expressed as

$$
\begin{gathered}
P(X)=\frac{1}{\sqrt{2 \pi \sigma_{X}^{2}}} e^{-\frac{\left(X-\mu_{X}\right)^{2}}{2 \sigma_{X}^{2}}}\left\{1+\sum_{n=1}^{\infty} A_{n} \frac{1}{\sqrt{n !}} H_{n}\left(\frac{X-\mu_{X}}{\sigma_{X}}\right)\right\}, \\
\mu_{\times}=\langle X\rangle, \quad \sigma_{X}^{2}=\left\langle\left(X-\mu_{X}\right)^{2}\right\rangle, \\
A_{n}=\left\langle\frac{1}{\sqrt{n !}} H_{n}\left(\frac{X-\mu_{X}}{\sigma_{X}}\right)\right\rangle,
\end{gathered}
$$

where $H_{n}(x)$ is the Hermite polynomial of $n$-th order. ${ }^{9}$

The observed data in the actual sound environment often contain fuzziness due to several factors such as limitations in the measuring instruments, permissible error tolerances in the measurement, and quantisation errors in digitising the observed data. Let the observed data comprising fuzzy observa- 